\title{
High-flow nasal therapy versus noninvasive ventilation in COPD patients with mild-to- moderate hypercapnic acute respiratory failure: study protocol for a noninferiority randomized clinical trial
}

\author{
Andrea Cortegiani $1^{*}$ (D) Federico Longhini ${ }^{2}$, Annalisa Carlucci ${ }^{3}$, Raffaele Scala ${ }^{4}$, Paolo Groff ${ }^{5}$, Andrea Bruni ${ }^{2}$, \\ Eugenio Garofalo ${ }^{2}$, Maria Rita Taliani ${ }^{5}$, Uberto Maccari ${ }^{4}$, Luigi Vetrugno ${ }^{6}$, Enrico Lupia ${ }^{7}$, Giovanni Misseri $^{1}$, \\ Vittoria Comellini ${ }^{8}$, Antonino Giarratano ${ }^{1}$, Stefano Nava ${ }^{8}$, Paolo Navalesi ${ }^{2}$ and Cesare Gregoretti ${ }^{1}$
}

\begin{abstract}
Background: Noninvasive ventilation (NIV) is indicated to treat respiratory acidosis due to exacerbation of chronic obstructive pulmonary disease (COPD). Recent nonrandomized studies also demonstrated some physiological effects of high-flow nasal therapy (HFNT) in COPD patients. We designed a prospective, unblinded, multicenter, randomized controlled trial to assess the noninferiority of HFNT compared to NIV with respect to the reduction of arterial partial pressure of carbon dioxide $\left(\mathrm{PaCO}_{2}\right)$ in patients with hypercapnic acute respiratory failure with mild-tomoderate respiratory acidosis.

Methods: We will enroll adult patients with acute hypercapnic respiratory failure, as defined by arterial pH between 7.25 and 7.35 and $\mathrm{PaCO}_{2} \geq 55 \mathrm{mmHg}$. Patients will be randomly assigned 1:1 to receive NIV or HFNT. NIV will be applied through a mask with a dedicated ventilator in pressure support mode. Positive end-expiratory pressure will be set at $3-5 \mathrm{cmH}_{2} \mathrm{O}$ with inspiratory support to obtain a tidal volume between 6 and $8 \mathrm{ml} / \mathrm{kg}$ of ideal body weight. HFNT will be initially set at a temperature of $37^{\circ} \mathrm{C}$ and a flow of $60 \mathrm{~L} / \mathrm{min}$. At 2 and $6 \mathrm{~h}$ we will assess arterial blood gases, vital parameters, respiratory rate, treatment intolerance and failure, need for endotracheal intubation, time spent under mechanical ventilation (both invasive and NIV), intensive care unit and hospital length of stay, and hospital mortality.

Based on an a error of $5 \%$ and a $\beta$ error of $80 \%$, with a standard deviation for $\mathrm{PaCO}_{2}$ equal to $15 \mathrm{mmHg}$ and a noninferiority limit of $10 \mathrm{mmHg}$, we computed a sample size of 56 patients. Considering potential drop-outs and nonparametric analysis, the final computed sample size was 80 patients (40 per group).

Discussion: HFNT is more comfortable than NIV in COPD patients recovering from an episode of exacerbation. If HFNT would not be inferior to NIV, HFNT could be considered as an alternative to NIV to treat COPD patients with mild-to-moderate respiratory acidosis.
\end{abstract}

Trial registration: ClinicalTrials.gov, NCT03370666. Registered on December 12, 2017.

Keywords: High-flow oxygen therapy through nasal cannula, Noninvasive ventilation, Chronic obstructive pulmonary disease, Carbon dioxide, Acute respiratory failure, Intensive care unit, Emergency department

\footnotetext{
* Correspondence: andrea.cortegiani@unipa.it

${ }^{1}$ Department of Surgical, Oncological and Oral Science (Di.Chir.On.S.), Section

of Anesthesia, Analgesia, Intensive Care and Emergency, Policlinico Paolo

Giaccone, University of Palermo, Via del vespro 129, 90127 Palermo, Italy

Full list of author information is available at the end of the article
}

(c) The Author(s). 2019 Open Access This article is distributed under the terms of the Creative Commons Attribution 4.0 International License (http://creativecommons.org/licenses/by/4.0/), which permits unrestricted use, distribution, and

reproduction in any medium, provided you give appropriate credit to the original author(s) and the source, provide a link to the Creative Commons license, and indicate if changes were made. The Creative Commons Public Domain Dedication waiver (http://creativecommons.org/publicdomain/zero/1.0/) applies to the data made available in this article, unless otherwise stated. 


\section{Background}

Severe exacerbation of chronic obstructive pulmonary disease (COPD) is defined as an acute worsening of chronic respiratory symptoms and decompensated respiratory acidosis [1]. In the case of hypercapnic acute respiratory failure (ARF), the sole optimization of standard medical therapy may fail in up to $74 \%$ of patients [2]. In addition to pharmacological therapy, recent guidelines recommend the use of noninvasive ventilation (NIV) in the absence of metabolic causes of acidosis [1, 3]. Indeed, NIV improves vital signs and gas exchanges [2, 4], increasing alveolar ventilation [5]. NIV also reduces the patient's effort [6], dyspnea [2, 7], the need for intubation $[2,8,9]$, the intensive care unit (ICU) length of stay [9] and the mortality rate $[2,9,10]$. However, there are several determinants for NIV success, including comfort related to the interface and patient-ventilator interaction $[4,11-18]$.

High-flow nasal therapy (HFNT) is a more recent treatment, which delivers a heated and humidified airoxygen mixture at high flow (up to $60 \mathrm{~L} / \mathrm{min}$ ) through large-bore nasal cannula $[19,20]$. Compared to standard oxygen therapy, HFNT washes out the anatomical dead space of the upper airway, generates a certain amount of expiratory pharyngeal pressure proportional to the flow, which increases the end-expiratory lung volume, slows the respiratory rate by decreasing the respiratory time [21], promotes the clearance of tracheobronchial secretions and reduces the inspiratory effort [22-24].

While a large body of evidence is already supporting the use of HFNT in critically ill patients with hypoxemic ARF [23, 25-28], few studies have evaluated its effectiveness in COPD patients [20]. In stable COPD patients, HFNT reduces the respiratory rate [29] and the arterial partial pressure of carbon dioxide $\left(\mathrm{PaCO}_{2}\right)[29,30]$, increases the tidal volume and the end-expiratory lung volume [29], and reduces the work of breathing and the rapid shallow breathing pattern [31, 32]. In patients recovering from an episode of hypercapnic ARF due to COPD exacerbation, the respiratory rate and drive significantly increase with standard oxygen at NIV discontinuation, while not with HFNT [33]. Furthermore, HFNT guaranteed optimal comfort [22, 34].

A recent prospective observational trial enrolling 92 patients with severe COPD exacerbation and moderate hypercapnic acute respiratory failure found no difference in 30-day mortality and the intubation rate between HNFT and NIV [34]. Currently, no randomized controlled trials have compared the efficacy of HFNT with that of NIV as first-line treatment in exacerbated COPD patients.

We conceived the present protocol in order to evaluate whether HFNT is noninferior to NIV in reducing $\mathrm{PaCO}_{2}$, in patients with an exacerbation of COPD and mild-to-moderate respiratory acidosis.

\section{Methods}

Study design

This is an investigator-initiated, unblinded, multicenter, noninferiority, randomized controlled trial. Randomization has been achieved using a computer-generated randomization sequence, generated by an independent investigator not otherwise involved in the trial, with an allocation ratio of 1:1 and with a permuted block method. A single randomization list was created for all participants. Allocation concealment has been maintained by using sequentially numbered sealed opaque envelopes. Each envelope contains the allocation of the patient to either control (NIV) or intervention (HFNT), with a unique patient identifier code. The randomization is based on a centralized phone call system. Due to the research design, neither the individual collecting data nor the patient can be blinded to treatment allocation.

The study was prospectively registered with ClinicalTrials.gov in December 2017 (identifier: NCT03370666). This protocol has been designed in accordance with the Standardized Protocol Items: Recommendations for Interventional Trials (SPIRIT) guidelines and checklist (Additional file 1). A schedule for enrolment, intervention and assessment (SPIRIT figure) is outlined in Fig. 1. A CONsolidated Standards of Reporting Trials (CONSORT) diagram is shown in Fig. 2.

\section{Setting}

The study will be conducted in the emergency department, ICU or respiratory unit of the following Italian centers: "Mater Domini" Hospital; "Magna Graecia" University, Catanzaro; Policlinico "Paolo Giaccone", University of Palermo; University Hospital of Udine; S. Donato Hospital, Arezzo; "S. Maria della Misericordia" Hospital, Perugia; and "Città della Salute e della Scienza" University Hospital, Torino. The study protocol has been prospectively approved by the local ethical committees. Further centers have recently submitted the study protocol for ethical committee approval.

\section{Study population}

We will consider eligible consecutive adult (i.e. $\geq 18$ years/old) patients with a diagnosis of COPD according to the GOLD criteria [1] admitted for an acute-onchronic respiratory failure due to exacerbation, with arterial $\mathrm{pH}$ between 7.25 and 7.35 and $\mathrm{PaCO}_{2} \geq 55$ $\mathrm{mmHg}$, to the emergency department or ICU or respiratory unit of the participating centers. Written informed consent will be acquired before the patient's inclusion. Patients will be excluded if they meet one or more of the following criteria: already received HFNT or NIV, prior to study enrolment; long-term domiciliary NIV; clinical instability, as defined by the need for vasopressors, acute coronary syndrome or life-threatening arrhythmias [35]; treatment refusal; agitation, as defined 


\begin{tabular}{|c|c|c|c|c|c|}
\hline \multirow[b]{3}{*}{ TIMEPOINT } & \multicolumn{5}{|c|}{ STUDY PERIOD } \\
\hline & \multirow{2}{*}{$\begin{array}{c}\text { Enrolment } \\
-t_{1}\end{array}$} & \multirow{2}{*}{$\begin{array}{c}\text { Allocation } \\
0\end{array}$} & \multicolumn{2}{|c|}{ Post-allocation } & \multirow{2}{*}{$\begin{array}{c}\text { Close-out } \\
\begin{array}{c}\text { Hospital } \\
\text { discharge }\end{array}\end{array}$} \\
\hline & & & 2 hours & 6 hours & \\
\hline \multicolumn{6}{|l|}{ ENROLMENT: } \\
\hline Eligibility screen & $X$ & & & & \\
\hline Informed consent & $X$ & & & & \\
\hline Allocation & & $x$ & & & \\
\hline \multicolumn{6}{|l|}{ INTERVENTIONS: } \\
\hline NIV & & & & $\rightarrow$ & \\
\hline HFOT & & & & & \\
\hline \multicolumn{6}{|l|}{ ASSESSMENTS: } \\
\hline Arterial Blood Gases & $x$ & & $x$ & $X$ & \\
\hline Dyspnea & $X$ & & $X$ & $\mathrm{X}$ & \\
\hline Interface discomfort & & & $X$ & $\mathrm{X}$ & \\
\hline Interface intolerance & & & $\mathrm{X}$ & $\mathrm{X}$ & \\
\hline Treatment failure & & & $\mathrm{X}$ & $\mathrm{X}$ & \\
\hline $\begin{array}{r}\text { Need for invasive } \\
\text { mechanical ventilation }\end{array}$ & & & $\mathrm{x}$ & $X$ & \\
\hline $\begin{array}{l}\text { Time spent under } \\
\text { mechanical ventilation }\end{array}$ & & & & & $\mathrm{X}$ \\
\hline ICU length of stay & & & & & $\mathrm{X}$ \\
\hline Hospital length of stay & & & & & $X$ \\
\hline Hospital mortality & & & & & $X$ \\
\hline
\end{tabular}

Fig. 1 A schedule for enrolment, intervention and assessment according to the Standard Protocol Items: Recommendations for Interventional Trials (SPIRIT) indications. HFNT high-flow nasal therapy through nasal cannula, ICU intensive care unit, NIV noninvasive ventilation

by Richmond Agitation Sedation Scale (RASS) score $\geq 2$, or lack of collaboration, as defined by Kelly Matthay score $\geq 5$ [35]; acute failure of more than two organs [35]; cardiac arrest; respiratory arrest deeming immediate intubation; recent trauma or bursts on the neck or face; pregnancy; consent withdrawal; and enrolment in other research protocols. The study flow chart is shown in Fig. 2.

\section{Intervention group}

Patients randomized to the intervention group will receive HFNT (Optiflow and MR850 or $\mathrm{AIrVO}_{2}{ }^{\mathrm{rm}}$; Fisher \& Paykel Healthcare, Auckland, New Zealand), initially set at $60 \mathrm{~L} / \mathrm{min}$ and a temperature of $37{ }^{\circ} \mathrm{C}$. In the case of discomfort, the flow and/or temperature will be downregulated to the most tolerated setting. In the HFNT group, a rescue NIV treatment will be allowed in the case of worsening of respiratory failure and/or treatment failure, before proceeding with an eventual endotracheal intubation and invasive mechanical ventilation.

\section{Control group}

Patients randomized to the control group will receive NIV applied through a total full-face or oro-nasal mask. The ventilator will be set in pressure support ventilation (PSV) mode, with positive end-expiratory pressure (PEEP) between 3 and $5 \mathrm{cmH}_{2} \mathrm{O}$. The inspiratory pressure support will be regulated in order to achieve a measured or estimated tidal volume equal to $6-8 \mathrm{ml} / \mathrm{kg}$ of ideal body weight [35]. The 
ASSESSED FOR ELIGIBILITY

COPD patients with an acute-on-chronic respiratory failure due to exacerbation

INCLUSION CRITERIA

Arterial $\mathrm{pH}$ in between 7.25 and 7.35 and $\mathrm{a} \mathrm{PaCO}_{2} \geq 55 \mathrm{mmHg}$

\begin{abstract}
EXCLUSION CRITERIA
HFNT or NIV prior the study enrolment;

long term domiciliary NIV; clinical

instability (need for vasopressors,

acute coronary syndrome or life-

threatening arrhythmias); treatment

refusal; agitation or lack of

collaboration; acute failure of two or

more organs; cardiac arrest; respiratory

arrest deeming immediate intubation;

recent trauma or bursts on the neck or

face; pregnancy; consent withdrawal; enrolment in other research protocols.
\end{abstract}

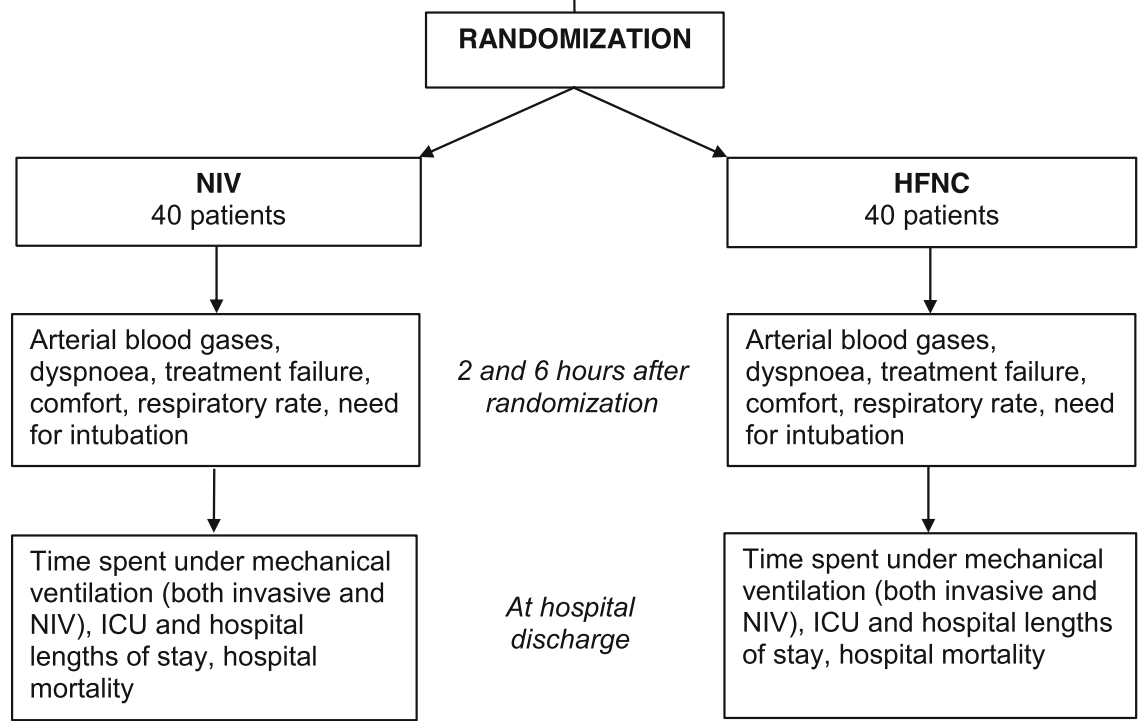

Fig. 2 Study flow chart according to CONsolidated Standards of Reporting Trials. COPD chronic obstructive pulmonary disease, HFNT high-flow nasal therapy through nasal cannula, ICU intensive care unit, NIV noninvasive ventilation, $\mathrm{PaCO}_{2}$ arterial partial pressure of carbon dioxide

treating physicians, based on local availability, will choose the ventilators used to deliver NIV.

In both groups, treatment other than ventilatory support will be supplied according to current guidelines [1]. Sedatives will be allowed to improve the patient's comfort and tolerance of the interfaces. The inspired oxygen fraction $\left(\mathrm{FiO}_{2}\right)$ will be set to maintain a peripheral saturation $\left(\mathrm{SpO}_{2}\right)$ target between 88 and 92\% [1]. In the case of treatment interruption due to any reason, patients will receive oxygen supply through a Venturi mask to achieve the same $\mathrm{SpO}_{2}$ target. During study interventions, patients will be monitored by at least continuous $\mathrm{SpO}_{2}$, electrocardiogram and noninvasive blood pressure.

\section{Study endpoints}

The primary endpoint of the study will be to evaluate whether HFNT is noninferior to NIV in reducing $\mathrm{PaCO}_{2}$, at $2 \mathrm{~h}$ after the group allocation, in patients with exacerbation of COPD and mild-to-moderate respiratory acidosis. The secondary endpoints will be: noninferiority of HFNT to NIV in reducing $\mathrm{PaCO}_{2}$ at $6 \mathrm{~h}$ after randomization; dyspnea score; treatment failure rate; discomfort related to the interface or to symptoms 
related to airway and mouth dryness; respiratory rate; need for endotracheal intubation; time spent under mechanical ventilation (both invasive and NIV); ICU and hospital length of stay; and hospital mortality.

\section{Data collection and outcome assessment}

For each patient, we will collect anthropometric data (age, gender, weight and height) and baseline characteristics (the Simplified Acute Physiology Score (SAPS II), the Kelly-Matthay score [36], the Charlson index [37] and the Richmond Agitation Sedation Scale (RASS) score [38]). Furthermore, soon before randomization we will record vital parameters (heart rate, blood pressure, breath frequency and temperature), the amount of secretions, the presence of dyspnea (as reported by the patient through the Borg scale) [39] and arterial blood gases (ABGs) at patient inclusion.

At 2 and $6 \mathrm{~h}$ after the patient's randomization to receive NIV or HFNT, we will collect all vital parameters, ABGs, the RASS score, the Kelly-Matthay score [36], the grade of dyspnea and the need for sedatives. Sedation will be allowed to increase tolerance to the treatment [40, 41]. Moreover, we will record: the settings of NIV and HFNT; the discomfort related to the interface, as assessed through a 11-point Numeric Rating Scale (NRS) $[15,42,43]$; and the intolerance to the interface due to flow, temperature, noise, claustrophobia, sweating, tightness, airway dryness, vomiting gastric distension, ocular irritation or skin breakdown, separately [42, $44,45]$.

We will also record: the rate of treatment intolerance, as defined by the inability of the patient in continuing NIV or HFNT treatment; the rate of treatment failure, as defined by the presence of $\mathrm{PaCO}_{2}$ worsening or reduction $<10 \mathrm{mmHg}$ from baseline assessment, or worsening or no improvement of dyspnea, or respiratory rate $>30$ breaths/min; need for endotracheal intubation; time spent under mechanical ventilation (both invasive and NIV); ICU and hospital length of stay; and hospital mortality.

The decision to intubate the patient will be taken by the physician in charge of the patient, in the case of the presence of one of the following criteria: respiratory arrest, respiratory apnea or pauses with loss of consciousness, severe agitation, bradycardia $(<50$ beats $/ \mathrm{min})$ with loss of consciousness, hemodynamic instability with systolic arterial pressure $<70 \mathrm{mmHg}$, need for invasive mechanical ventilation due to worsening in arterial blood gases and $\mathrm{pH}$, need for suctioning due to unmanageable secretions or intolerance to all of the interfaces (including shift of treatment to NIV in the HFNT group).

Data will be collected on a dedicated case report form that will be sent to the data manager in de-identified form. The data manager will be responsible for entering data into a dedicated database, to code and store them. Treatment allocation will also be processed in order for the independent investigator in charge of the statistical analysis to be blinded.

\section{Statistical analysis}

For the purpose of the study, we computed a sample size of 56 patients, given an $\alpha$ error of $5 \%$ (one-sided) and a $\beta$ error of $80 \%$, with a standard deviation for the primary outcome equal to $15 \mathrm{mmHg}$ and a noninferiority limit of $10 \mathrm{mmHg}$ [35]. After considering potential drop-outs (30\%) [35] and an increase in sample size for nonparametric analysis (15\%), the final computed sample size was 80 patients (40 per group).

An independent investigator, blinded to treatment allocation, will undertake all statistical analyses on an intention-to-treat basis. After checking the Gaussian distribution, continuous data will be presented as mean (standard deviation) or median (25th-75th IQR).

Categorical data will be expressed as count and percentage. Continuous data will be compared by the Mann-Whitney $U$ test or the Student $t$ test, and categorical data by the chi-square test or the Fisher exact test [46]. The Kruskal-Wallis test will be used to check between-group differences at baseline, $2 \mathrm{~h}$ and $6 \mathrm{~h}$. Friedman's test or repeated-measures ANOVA will be used to check within-group differences at different time points. $p<0.05$ will be considered significant.

\section{Study organization}

The steering committee consists of two principal investigators (AC, FL) plus six members with recognized expertise on NIV/HFNT in COPD patients. The steering committee invited other centers based on expertise and availability of study patients and materials. Each participating center has a local principal investigator in charge. They act as the guarantors of the data, and are responsible for data management and storage in their centers. Local principal investigators also communicate with the principal investigators and transfer patient data in an anonymized form on standard case report forms; in the case of logistic difficulties or study doubts, the local investigators can ask for immediate phone call support from principal investigators, to achieve adequate participant enrolment. Data monitoring will be done by an ndependent investigator serving as the data manager. This will be done by means of queries on the final databases, after collecting the case report forms from enrolling centers, to check for abnormalities and inconsistencies.

\section{Discussion}

HFNT is increasingly used in different settings [23, 25-27]. Some studies indicate that HFNT has physiological 
advantages in both stable and exacerbated COPD patients [20]; in particular, in patients recovering from an episode of hypercapnic ARF due to COPD exacerbation, HFNT reduces the respiratory effort and rate no differently from NIV, while it maintains similar gas exchanges and improved comfort [22]. A recent observational trial found no difference between NIV and HNFT in terms of 30-day mortality and intubation rate in severe exacerbation of COPD [34].

Currently, NIV is strongly suggested as standard treatment for exacerbated COPD patients with respiratory acidosis [3]. However, NIV is affected by several drawbacks such as reduced comfort and poor patient-ventilator interaction and synchrony, which is often difficult to be recognized and managed $[4,11-13,16]$. We therefore design this randomized controlled trial to assess the noninferiority of HFNT in reducing the $\mathrm{PaCO}_{2}$, as compared to NIV. In fact, whenever the trial demonstrates a noninferiority of HFNT compared to NIV, the physician may use HFNT as an alternative ventilatory support in exacerbated COPD patients with mild-to-moderate respiratory acidosis. This would also allow the possibility to use another support with a different interface in a rotational strategy to improve the patient's comfort and tolerance to the treatment $[47,48]$. This trial has limitations. First, blinding is not possible due to the nature of the interventions. Second, although recruitment centers have established experience in the use of ventilator support in this patient population, different practices in the use of both NIV and HNFT may influence the effect of the interventions. However, recruitment centers have established experience in the use of ventilator support in this patient population. Third, the study is a noninferiority trial with a physiologic outcome, leaving uncertainty on stronger patient-related outcomes. It is anticipated that the results of this study will be used to design further randomized clinical trials powered to assess major clinical outcomes.

\section{Trial status}

The study is currently recruiting patients. The protocol was approved by the Ethics Committee of the coordination center on 24 October 2017 ("Comitato Etico sezione Area Centro- $\left.\mathrm{N}^{\circ} 245^{\prime \prime}\right)$. The first patient was enrolled on 13 March 2018. Recruitment is expected to be completed within June 2020 .

\section{Additional file}

Additional file 1: SPIRIT 2013 Checklist: Recommended items to address in a clinical trial protocol and related documents (DOC $122 \mathrm{~kb}$ )

\section{Abbreviations}

ABG: Arterial blood gas; ARF: Acute respiratory failure; CONSORT: CONsolidated Standards of Reporting Trials; COPD: Chronic obstructive pulmonary disease; HFNT: High-flow nasal therapy;
NIV: Noninvasive ventilation; NRS: Numeric Rating Scale; $\mathrm{PaCO}_{2}$ : Arterial partial pressure of carbon dioxide; PEEP: Positive end-expiratory pressure; RASS: Richmond Agitation Sedation Scale; SAPS II: Simplified Acute Physiology Score; SPIRIT: Standard Protocol Items: Recommendations for Interventional Trials

\section{Acknowledgments \\ Not applicable.}

\section{Authors' contributions}

All authors participated in the conception and design of the study. All authors are responsible for the patients' enrolment and data acquisition in their institution. ACo, FL, PN and CG participated in manuscript drafting. All authors approved the final manuscript version, and accounted for all aspects of the work in ensuring that questions related to the accuracy and integrity of any part of the work were appropriately investigated and resolved.

\section{Funding}

No funding has been received for this trial. Some centers receive material support (devices and cannula) from Fisher \& Paykel, which has no role in protocol design, data management, interpretation and analysis.

\section{Availability of data and materials}

The authors will share all of the individual participant data collected during the trial, after de-identification, to researchers who provide a methodologically sound proposal.

\section{Ethics approval and consent to participate}

The Ethics Committee of the coordinating center approved the study protocol on 24 October 2017 ("Comitato Etico sezione Area Centro-N²45"). The local ethics committees or institutional review boards of participating centers have approved the study protocol. Consent to participate in the trial will be obtained from every patient according to national regulation.

\section{Consent for publication}

Not applicable.

\section{Competing interests}

Prof. Stefano Nava's institution received an unrestricted research grant from Fisher and Paykel. Prof. Paolo Navalesi's institution received funding from Maquet Critical Care, Draeger and Intersurgical S.p.A.; he received honoraria/ speaking fees from Maquet Critical Care, Orionpharma, Philips, Resmed, Merck Sharp \& Dome and Novartis; and he discloses that he contributed to the development of the helmet Next, whose license for patent belongs to Intersurgical S.P.A., and receives royalties for that invention.

Prof. Federico Longhini and Prof. Paolo Navalesi contributed to the development of a device not discussed in the present study whose patent is in progress (European Patent application number EP20170199831). Dr Paolo Groff received fees for lectures from Aspen Pharmaceuticals and Menarini Pharmaceuticals (not relevant to this protocol). Prof. Cesare Gregoretti received fees for lectures or consultancies from Philips, Resmed, Vivisol, OrionPharma and Origin (not relevant to this protocol). The remaining authors disclose that they have no conflicts of interest.

\section{Author details}

${ }^{1}$ Department of Surgical, Oncological and Oral Science (Di.Chir.On.S.), Section of Anesthesia, Analgesia, Intensive Care and Emergency, Policlinico Paolo Giaccone, University of Palermo, Via del vespro 129, 90127 Palermo, Italy. ${ }^{2}$ Intensive Care Unit, Department of Medical and Surgical Sciences, University Hospital Mater Domini, Magna Graecia University, Catanzaro, Italy.

${ }^{3}$ Pulmonary Rehabilitation Unit, Istituti Clinici Scientifici Maugeri, Pavia, Italy. ${ }^{4}$ Pulmonology and Respiratory Intensive Care Unit, S. Donato Hospital, Arezzo, Italy. ${ }^{5}$ Emergency Department, "S. Maria della Misericordia" Hospital, Perugia, Italy. ${ }^{6}$ Department of Anaesthesia and Intensive Care, University of Udine, Udine, Italy. ${ }^{7}$ Emergency Department, "Città della Salute e della Scienza" University Hospital, Torino, Italy. ${ }^{8}$ Department of Clinical, Integrated and Experimental Medicine (DIMES), Respiratory and Critical Care Unit, S. Orsola-Malpighi Hospital, Alma Mater University, Bologna, Italy. 
Received: 29 March 2019 Accepted: 10 June 2019

\section{Published online: 22 July 2019}

\section{References}

1. Vogelmeier CF, Criner GJ, Martinez FJ, Anzueto A, Barnes PJ, Bourbeau J, et al. Global Strategy for the Diagnosis, Management, and Prevention of Chronic Obstructive Lung Disease 2017 Report: GOLD Executive Summary. Eur Respir J. 2017;49:1700214.

2. Plant PK, Owen JL, Elliott MW. Early use of non-invasive ventilation for acute exacerbations of chronic obstructive pulmonary disease on general respiratory wards: a multicentre randomised controlled trial. Lancet. 2000; 355:1931-5.

3. Rochwerg B, Brochard L, Elliott MW, Hess D, Hill NS, Nava S, et al. Official ERS/ATS clinical practice guidelines: noninvasive ventilation for acute respiratory failure. Eur Respir J. 2017;50:1602426 European Respiratory Society.

4. Gregoretti C, Pisani L, Cortegiani A, Ranieri VM. Noninvasive ventilation in critically ill patients. Crit Care Clin. 2015;31:435-57.

5. Diaz O, Iglesia R, Ferrer M, Zavala E, Santos C, Wagner PD, et al. Effects of noninvasive ventilation on pulmonary gas exchange and hemodynamics during acute hypercapnic exacerbations of chronic obstructive pulmonary disease. Am J Respir Crit Care Med. 1997;156:1840-5.

6. Appendini L, Patessio A, Zanaboni S, Carone M, Gukov B, Donner CF, et al. Physiologic effects of positive end-expiratory pressure and mask pressure support during exacerbations of chronic obstructive pulmonary disease. Am J Respir Crit Care Med. 1994;149:1069-76.

7. Bott J, Carroll MP, Conway JH, Keilty SE, Ward EM, Brown AM, et al. Randomised controlled trial of nasal ventilation in acute ventilatory failure due to chronic obstructive airways disease. Lancet. 1993;341:1555-7.

8. Brochard L, Mancebo J, Wysocki M, Lofaso F, Conti G, Rauss A, et al. Noninvasive ventilation for acute exacerbations of chronic obstructive pulmonary disease. N Engl J Med. 1995;333:817-22.

9. Kramer N, Meyer TJ, Meharg J, Cece RD, Hill NS. Randomized, prospective trial of noninvasive positive pressure ventilation in acute respiratory failure. Am J Respir Crit Care Med. 1995;151:1799-806.

10. Cortegiani A, Russotto V, Antonelli M, Azoulay E, Carlucci A, Conti G, et al. Ten important articles on noninvasive ventilation in critically ill patients and insights for the future: a report of expert opinions. BMC Anesthesiol. 2017;17:122.

11. Longhini F, Colombo D, Pisani L, Idone F, Chun P, Doorduin J, et al. Efficacy of ventilator waveform observation for detection of patient-ventilator asynchrony during NIV: a multicentre study. ERJ Open Res. 2017;3:000752017 European Respiratory Society.

12. Nava S, Hill N. Non-invasive ventilation in acute respiratory failure. Lancet. 2009;374:250-9.

13. Bruni A, Garofalo E, Pelaia C, Messina A, Cammarota G, Murabito P, et al. Patient-ventilator asynchrony in adult critically ill patients. Minerva Anestesiol. 2019;85(6):676-88.

14. Cortegiani A, Madotto F, Gregoretti C, Bellani G, Laffey JG, Pham T, et al. Immunocompromised patients with acute respiratory distress syndrome: secondary analysis of the LUNG SAFE database. Crit Care. 2018;22:157.

15. Longhini F, Liu L, Pan C, Xie J, Cammarota G, Bruni A, et al. Neurallyadjusted ventilatory assist for noninvasive ventilation via a helmet in subjects with COPD exacerbation: a physiologic study. Respir Care. 2019; 64(5):582-9.

16. Garofalo E, Bruni A, Pelaia C, Liparota L, Lombardo N, Longhini F, et al. Recognizing, quantifying and managing patient-ventilator asynchrony in invasive and noninvasive ventilation. Expert Rev Respir Med. 2018;12:557-67.

17. Liu L, Xia F, Yang Y, Longhini F, Navalesi P, Beck J, et al. Neural versus pneumatic control of pressure support in patients with chronic obstructive pulmonary diseases at different levels of positive end expiratory pressure: a physiological study. Crit Care. 2015;19:244

18. Carlucci A, Schreiber A, Mattei A, Malovini A, Bellinati J, Ceriana P, et al. The configuration of bi-level ventilator circuits may affect compensation for non-intentional leaks during volume-targeted ventilation. Intensive Care Med. 2013;39:59-65.

19. Cortegiani A, Accurso G, Mercadante S, Giarratano A, Gregoretti C. High flow nasal therapy in perioperative medicine: from operating room to general ward. BMC Anesthesiol. 2018;18:166.

20. Spoletini G, Cortegiani A, Gregoretti C. Physiopathological rationale of using high-flow nasal therapy in the acute and chronic setting: a narrative review. Trends Anaesth Crit Care. 2019. https://doi.org/10.1016/j.tacc.2019.02.001.
21. Mundel T, Feng S, Tatkov S, Schneider H. Mechanisms of nasal high flow on ventilation during wakefulness and sleep. J Appl Physiol (1985). 2013;114:1058-65.

22. Longhini F, Pisani L, Lungu R, Comellini V, Bruni A, Garofalo E, et al. HighFlow Oxygen Therapy After Noninvasive Ventilation Interruption in Patients Recovering From Hypercapnic Acute Respiratory Failure: A Physiological Crossover Trial. Crit Care Med. 2019. https://doi.org/10.1097/CCM. 0000000000003740

23. Renda T, Corrado A, Iskandar G, Pelaia G, Abdalla K, Navalesi P. High-flow nasal oxygen therapy in intensive care and anaesthesia. Br J Anaesth. 2018;120:18-27.

24. Pisani L, Fasano L, Corcione N, Comellini V, Musti MA, Brandao M, et al. Change in pulmonary mechanics and the effect on breathing pattern of high flow oxygen therapy in stable hypercapnic COPD. Thorax. 2017;72:373-5.

25. Russotto V, Cortegiani A, Raineri SM, Gregoretti C, Giarratano A. Respiratory support techniques to avoid desaturation in critically ill patients requiring endotracheal intubation: a systematic review and meta-analysis. J Crit Care. 2017:41:98-106.

26. Cortegiani A, Crimi C, Sanfilippo F, Noto A, Di Falco D, Grasselli G, et al. High flow nasal therapy in immunocompromised patients with acute respiratory failure: a systematic review and meta-analysis. J Crit Care. 2019:50:250-6.

27. Rochwerg B, Granton D, Wang DX, Helviz Y, Einav S, Frat JP, et al. High flow nasal cannula compared with conventional oxygen therapy for acute hypoxemic respiratory failure: a systematic review and meta-analysis. Intensive Care Med. 2019;45:563-72.

28. Cortegiani A, Crimi C, Noto A, Helviz Y, Giarratano A, Gregoretti C, et al. Effect of high-flow nasal therapy on dyspnea, comfort, and respiratory rate. Crit Care. 2019;23:201.

29. Fraser JF, Spooner AJ, Dunster KR, Anstey CM, Corley A. Nasal high flow oxygen therapy in patients with COPD reduces respiratory rate and tissue carbon dioxide while increasing tidal and end-expiratory lung volumes: a randomised crossover trial. Thorax. 2016;71:759-61.

30. Vogelsinger $H$, Halank M, Braun S, Wilkens H, Geiser T, Ott S, et al. Efficacy and safety of nasal high-flow oxygen in COPD patients. BMC Pulm Med. 2017; 17:143

31. Braunlich J, Kohler M, Wirtz H. Nasal highflow improves ventilation in patients with COPD. Int J Chron Obstruct Pulmon Dis. 2016;11:1077-85.

32. Fricke K, Tatkov S, Domanski U, Franke K-J, Nilius G, Schneider H. Nasal high flow reduces hypercapnia by clearance of anatomical dead space in a COPD patient. Respir Med Case Rep. 2016;19:115-7.

33. Di Mussi R, Spadaro S, Stripoli T, Volta CA, Trerotoli P, Pierucci P, et al. Highflow nasal cannula oxygen therapy decreases postextubation neuroventilatory drive and work of breathing in patients with chronic obstructive pulmonary disease. Crit Care. 2018;22:180.

34. Lee MK, Choi J, Park B, Kim B, Lee SJ, Kim S-H, et al. High flow nasal cannulae oxygen therapy in acute-moderate hypercapnic respiratory failure. Clin Respir J. 2018;12:2046-56.

35. Pisani L, Mega C, Vaschetto R, Bellone A, Scala R, Cosentini R, et al. Oronasal mask versushelmet in acute hypercapnic respiratory failure. Eur Respir J. 2015;45:691-9 European Respiratory Society.

36. Kelly BJ, Matthay MA. Prevalence and severity of neurologic dysfunction in critically ill patients. Influence on need for continued mechanical ventilation. Chest. 1993;104:1818-24.

37. Quan H, Li B, Couris CM, Fushimi K, Graham P, Hider P, et al. Updating and validating the Charlson comorbidity index and score for risk adjustment in hospital discharge abstracts using data from 6 countries. Am J Epidemiol. 2011;173:676-82.

38. Sessler CN, Gosnell MS, Grap MJ, Brophy GM, O'Neal PV, Keane KA, et al. The Richmond Agitation-Sedation Scale: validity and reliability in adult intensive care unit patients. Am J Respir Crit Care Med. 2002;166:1338-44.

39. Kendrick KR, Baxi SC, Smith RM. Usefulness of the modified 0-10 Borg scale in assessing the degree of dyspnea in patients with COPD and asthma. J Emerg Nurs. 2000;26:216-22.

40. Hilbert G, Navalesi P, Girault C. Is sedation safe and beneficial in patients receiving NIV? Yes. Intensive Care Med. 2015;41:1688-91.

41. Costa R, Navalesi P, Cammarota G, Longhini F, Spinazzola G, Cipriani F, et al. Remifentanil effects on respiratory drive and timing during pressure support ventilation and neurally adjusted ventilatory assist. Respir Physiol Neurobiol. 2017;244:10-6.

42. Maggiore SM, Idone FA, Vaschetto R, Festa R, Cataldo A, Antonicelli F, et al. Nasal high-flow versus Venturi mask oxygen therapy after extubation. Effects on oxygenation, comfort, and clinical outcome. Am J Respir Crit Care Med. 2014;190:282-8. 
43. Cammarota G, Longhini F, Perucca R, Ronco C, Colombo D, Messina A, et al. New setting of neurally adjusted ventilatory assist during noninvasive ventilation through a helmet. Anesthesiology. 2016;125:1181-9.

44. Antonelli M, Conti G, Pelosi P, Gregoretti C, Pennisi MA, Costa R, et al. New treatment of acute hypoxemic respiratory failure: noninvasive pressure support ventilation delivered by helmet-a pilot controlled trial. Crit Care Med. 2002;30:602-8.

45. Gregoretti C, Confalonieri M, Navalesi P, Squadrone V, Frigerio P, Beltrame F, et al. Evaluation of patient skin breakdown and comfort with a new face mask for non-invasive ventilation: a multi-center study. Intensive Care Med. 2002;28:278-84.

46. Zhang Z. Univariate description and bivariate statistical inference: the first step delving into data. Ann Transl Med. 2016;4:91.

47. Vaschetto R, Longhini F, Persona P, Ori C, Stefani G, Liu S, et al. Early extubation followed by immediate noninvasive ventilation vs. standard extubation in hypoxemic patients: a randomized clinical trial. Intensive Care Med. 2019;45:62-71.

48. Nava S, Navalesi P, Carlucci A. Non-invasive ventilation. Minerva Anestesiol. 2009:75:31-6.

\section{Publisher's Note}

Springer Nature remains neutral with regard to jurisdictional claims in published maps and institutional affiliations.

Ready to submit your research? Choose BMC and benefit from:

- fast, convenient online submission

- thorough peer review by experienced researchers in your field

- rapid publication on acceptance

- support for research data, including large and complex data types

- gold Open Access which fosters wider collaboration and increased citations

- maximum visibility for your research: over $100 \mathrm{M}$ website views per year

At $\mathrm{BMC}$, research is always in progress.

Learn more biomedcentral.com/submissions 